\title{
Surface-Based Texture and Morphological Analysis Detects Subtle Cortical Dysplasia
}

\author{
Pierre Besson $^{1}$, Neda Bernasconi ${ }^{1}$, Olivier Colliot ${ }^{2}$, Alan Evans ${ }^{1}$, \\ and Andrea Bernasconi ${ }^{1}$ \\ ${ }^{1}$ McConnell Brain Imaging Centre, Montreal Neurological Institute, \\ Montreal, Canada \\ ${ }^{2}$ Cognitive Neuroscience and Brain Imaging Laboratory, CNRS UPR 640-LENA, \\ Université Pierre et Marie Curie - Paris 6, \\ Hôpital de la Pitié-Salpêtrière, Paris, France
}

\begin{abstract}
Focal cortical dysplasia (FCD), a malformation of cortical development, is an important cause of pharmacoresistant epilepsy. Small FCD lesions are difficult to distinguish from normal cortex and remain often overlooked on radiological MRI inspection. This paper presents a method to detect small FCD lesions on T1-MRI relying on surface-based features that model their textural and morphometric characteristics. The automatic detection was performed by a two step classification. First, a vertex-wise classifier based on a neural-network bagging trained on manual labels. Then, a cluster-wise classification designed to remove false positive clusters. The method was tested on 19 patients with small FCD. At the first classification step, 18/19 (95\%) lesions were detected. The second classification step kept 13/19 (68\%) lesions and decreased efficiently the amount of false positive. This new approach may assist the presurgical evaluation of patients with intractable epilepsy, especially those with unremarkable MRI findings.
\end{abstract}

\section{Introduction}

Malformations of cortical development have been increasingly recognized as an important cause of pharmacoresistant epilepsy. Focal cortical dysplasia (FCD) [1, a malformation due to abnormal neuroglial proliferation, is the most frequent form in patients with intractable epilepsy [2]. Epilepsy surgery, consisting in the removal of the FCD lesion, is an effective treatment for these patients and magnetic resonance images (MRI) play a pivotal role in presurgical evaluation 3 .

Previous automated image analysis techniques to detect FCD on MRI relied on various types of voxel-wise analyses [4,56. In particular, computational models of FCD characteristics were developed to highlight the lesion [7] and a Bayesian classifier used for lesion detection [4. While these approaches successfully identified FCD in a majority of patients, most of the lesions included in these studies were detected on routine radiological evaluation. On the other hand, the detection of small FCD lesions, overlooked in more than $80 \%$ of cases [8], is a much more challenging task and has never been addressed.

D. Metaxas et al. (Eds.): MICCAI 2008, Part I, LNCS 5241, pp. 645-652, 2008

(C) Springer-Verlag Berlin Heidelberg 2008 
Recently, robust and automatic methods have been developed to reconstruct the inner and outer cortical surfaces 910]. These techniques have enabled the identification of subtle variations in cortical thickness in the healthy [11] and diseased brain [12, and were also used for morphometric analysis of the cortex [13]. Moreover, cortical matching techniques align corresponding cortices across individuals using morphological measurement such as specific sulci [14] or cortical surface curvature [15, thus allowing a precise vertex-wise comparison.

This paper presents a new method for detecting small FCD lesions on T1weighted MRI that relies on surface-based features. To that purpose, we modeled morphological and textural characteristics of FCD at each vertex of the cortical surface. To our knowledge, this is the first application of surface-based analysis to the detection of cortical malformations.

\section{Methods}

\subsection{Image Acquisition and Preprocessing}

3D MR images were acquired on a $1.5 \mathrm{~T}$ scanner using a T1-fast field echo sequence $(\mathrm{TR}=18, \mathrm{TE}=10,1$ acquisition average pulse sequence, flip angle=30, matrix size $=256 \times 256, \mathrm{FOV}=256$, thickness $=1 \mathrm{~mm})$ with an isotropic voxel size of $1 \mathrm{~mm} 3$. All images underwent automated correction for intensity non-uniformity and intensity standardization [16], automatic registration into stereotaxic space [17], automatic tissue classification [18] and brain extraction.

\subsection{FCD Features Extraction}

Five features were extracted from the MR images. These features correspond to morphometric and textural characteristics specific to small FCD lesions: cortical thickening, blurred grey matter (GM) / white matter (WM) transition, hyperintense T1 signal, deep beneath the outer cortical surface and located at concavely curved cortical surface [8].

In each hemisphere, the inner and outer-cortical surfaces were computed using the CLASP (Constrained Laplacian Anatomical Segmentation using Proximities) algorithm [9]. The inner-cortical surface was extracted by inflating a sphere polygon model to the boundary between GM and WM. The outer-cortical surface was obtained by expanding the inner-cortical surface to match the boundary between GM and cerebrospinal fluid (CSF). These two surfaces are formed of 81920 corresponding vertices.

The cortical thickness was measured using the t-link method, defined as the distance between corresponding vertices [19].

The blurred $W M / G M$ interface was modeled by applying a gradient operator on the MR image. The gradient magnitude was then interpolated at each vertex of the inner cortical surface to obtain the gradient surface map (Fig. 11A).

To model hyperintensity of the lesion with respect to healthy cortex, we constructed three equidistant intra-cortical surfaces by placing three uniformly 


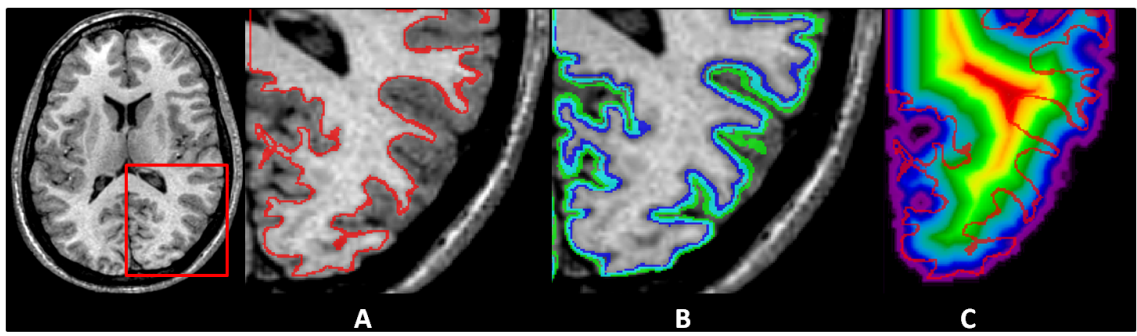

Fig. 1. Projection of the features on cortical surfaces. A. Inner cortical surface onto which the gradient was projected to model the blurred WM/GM transition. B. Three equidistantly placed intra-cortical surfaces between WM and GM surfaces on which T1 signal intensity was projected and averaged. C. Depth calculated as the distance between the WM surface and the boundary of the cortical mask.

spaced vertices between linked vertices of inner and outer cortical surfaces. The intensity of the underlying MR image was then interpolated at each vertex of the intra cortical surfaces. The intensity feature was modeled by the mean intensity of the three corresponding vertices of the intra-cortical surfaces (Fig. 11B).

The depth was defined as the shortest distance between each vertex of the WM surface and the boundary of the brain mask obtained with the brain extraction tool (BET) (Fig. 11C).

The curvature was calculated at each vertex using area-minimizing flows to define a deviation from the cortical surface to a sphere [15].

\subsection{Vertex-Based Analysis}

In the normal brain, feature values vary depending on the anatomical location. To take into account these regional variations, we proposed to use a vertex-based analysis (VBA) of the features by applying vertex-wise comparison between a group of healthy controls and a given patient. VBA included the following steps: 1) blurring of the features using a 5 mm FWHM Gaussian surface kernel [19]; 2) registration of the surface features to a template [15] 3) computation of the mean and standard deviation (SD) at each vertex within the group of healthy controls; 4) deviation from normal is obtained using vertex-wise z-score transform for each patient with respect to the healthy controls mean and SD. We computed the VBA on cortical thickness, gradient, intensity and depth maps.

To classify small FCD lesions, we used the following features: VBA of the gradient and VBA of the intensity (to model the textural characteristics of the lesions); cortical surface curvature and depth, VBA of the cortical thickness and VBA of depth (to model the morphometric characteristics of the lesions).

\subsection{Automatic FCD Detection}

The automatic detection was performed using two classification steps. The first step was designed to recognize lesional vertices with the highest detection rate. 
The purpose of the second step was to remove the false positives (FP) generated by the first classifier using a cluster-wise classification.

Lesions were manually segmented on 3D MRI by trained raters and interpolated at each vertex of the cortical surfaces. Since the small FCD lesions are difficult to distinguish on T1-MRI, the rater made use of other image sequences such as T2, proton density (PD) and fluid-attenuated inversion recovery (FLAIR), when available. The spatial extent of the lesions being difficult to define [20], the labels were considered as silver standard.

Vertex-Wise Classification. For vertex-wise classification, we chose to use four layer feed forward neural networks with the following number of neurons in each layer: (6-4-4-1); tan-sigmoid function was used at each neuron and the output node resulted in a number between 0 and 1 representing the probability of being lesional. To avoid over fitting, we used a cross-validation method to optimize the nets. From all patients, we obtained a database constituted of about $2.8 \cdot 10^{2} 6$ non-lesional and 1841 lesional instances in which we randomly picked 200 vertices (80 lesional, 120 non-lesional) to construct the training set and 200 different vertices (80 lesional, 120 non-lesional) for the validation set. The neural network was optimized on the training set until the error on validation set started increasing. To avoid poorly performing nets, we used a bagging approach: we created 200 nets and kept only the best 100 (i.e. having the lowest validation error). The proportion of lesional instances in the training and validation sets and the ratio of discarded nets were ad hoc choices obtained from experiments. The final output of the networks bagging was the average of the 100 nets.

The lesional probability maps obtained from the classifier were binarized by thresholding them to keep a high detection rate and an acceptable amount of false positives $(\mathrm{FP})$.

Cluster-Wise Classification. Clusters (defined by the 6-connected neighbours of the triangulated cortical surface) generated by the vertex-wise classifier were further separated into FP or lesional based on their global features as explained below.

Each cluster was characterized by 13 features: size, mean and SD of the 6 features used for vertex-wise classification. The classification step was performed using a fuzzy k-Nearest Neighbor classifier (fkNN) that determines the membership value to the lesional class. A leave-one-out scheme was used to construct the training set. The clusters of all the patients but one were compared to the corresponding manual label and identified as FP or lesional. They constituted the training set for the classification of the excluded patient. On the other hand, the classification of the healthy controls was performed using a training set formed by all patients.

To find the best trade-off between the lesional detection rate and the amount of FP in healthy controls, we plotted the lesional membership value threshold against the detection rate and the number of false positives clusters in controls. 


\section{Experiment and Results}

\subsection{Subjects}

We studied 41 consecutive patients with FCD. The volume of the lesions ranged from 128 to $94620 \mathrm{~mm} 3$ (mean $\pm \mathrm{SD}=7731 \pm 14891 \mathrm{~mm} 3$ ).

Using an entropy index based on their size and visibility on routine clinical MRI examination ( $89 \%$ of small FCD - volume smaller than $3093 \mathrm{~mm}^{3}$ - were overlooked, as opposed to $0 \%$ of FCD larger than $3093 \mathrm{~mm}^{3}$ ) [8, 19 patients had a lesion defined as small and therefore were included in the study (mean age $=24.9 \pm 10.9$ ). Their mean volume was $1380 \pm 808 \mathrm{~mm} 3$ (range: $128-3093$ mm3), 17/19 (89\%) had been overlooked on routine clinical MRI examination. The extent of the manual labels on the cortical surface was $96 \pm 66$ vertices (range: 14 - 236).

We used 48 healthy controls (mean age $=27.3 \pm 7.8$ ) to construct the VBA models 11 additional healthy controls (mean age $=29.8 \pm 10.1$; 59 healthy controls in total) for the evaluation of the FP rate generated by the classification steps.

\section{$3.2 \quad$ Results}

The results of the two classifiers are summarized in Table 1.

For vertex-wise classification, we set the threshold to 0.87 and detected the FCD in 18/19 (95\%) patients. The size of the lesional clusters was $39.9 \pm 58.9$ vertices (range: 1 - 226). On average, $23.1 \pm 19.8$ (range: 1 - 79) FP clusters were generated in all patients. Their size was $7.4 \pm 11.5$ vertices (range: 1 - 99 vertices). In healthy controls, the vertex-wise classifier produced $7.1 \pm 5.7 \mathrm{FP}$ clusters (size: $10.0 \pm 9.9$ vertices) in 57/59 (96\%) healthy controls.

Table 1. Summary of the results obtained by the two step classification. Detection rate and size (mean $\pm \mathrm{SD}$ vertices) of the lesional clusters. Size and amount (number of FP clusters) of false positive clusters in patients and in healthy controls (HC).

\begin{tabular}{|c|c|c|c|c|c|c|}
\hline \multirow{2}{*}{ Step } & \multicolumn{2}{|c|}{ Lesional Clusters } & \multicolumn{2}{c|}{$\begin{array}{c}\text { False positive clusters in } \\
\text { patients }\end{array}$} & \multicolumn{2}{c|}{ False Potive Clusters in HC } \\
\cline { 2 - 7 } & Detection Rate & Size & Size & Quantity & Size & Quantity \\
\hline $\begin{array}{c}\text { Vertex-wise } \\
\text { Classifier }\end{array}$ & $18 / 19(95 \%)$ & $39.9 \pm 58.9$ & $7.4 \pm 11.5$ & $23.1 \pm 19.8$ & $10.0 \pm 9.9$ & $7.1 \pm 5.7$ \\
\hline $\begin{array}{c}\text { Cluster-wise } \\
\text { Classifier }\end{array}$ & $13 / 19(68 \%)$ & $82.2 \pm 71.6$ & $30.2 \pm 25.2$ & $2.8 \pm 2.2$ & $27.2 \pm 21.3$ & $1.4 \pm 0.8$ \\
\hline
\end{tabular}

For the cluster-wise classification, we chose $\mathrm{k}=20$ for the fkNN classifier because of the large number of FP clusters relatively to the lesional clusters in patients. We chose a lesional membership value threshold of 0.13 (Figure 2) to achieve the best trade-off between FCD detection and the amount of FP in healthy controls. Using these parameters, $13 / 19$ (68\%) lesions were detected. The size of the lesional clusters was $82.2 \pm 71.6$ vertices (range: 8 - 126 vertices). 


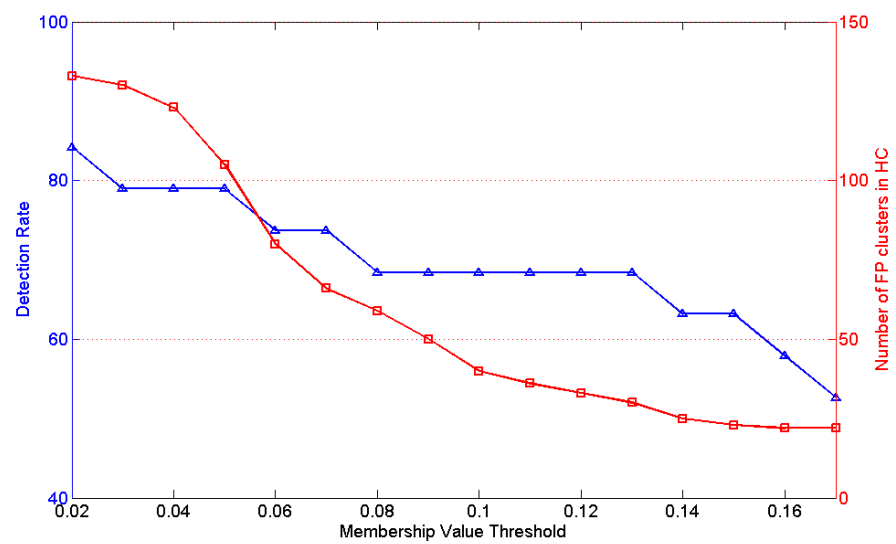

Fig. 2. Detection rate and amount of FP clusters in healthy controls (HC) (y-axis) plotted against the membership value threshold. We set the threshold to 0.13 .

In 12/19 (63\%) patients, we found $2.8 \pm 2.2 \mathrm{FP}$ clusters (range: 1 - 8) and their size was $30.2 \pm 25.2$ vertices (range: 1 - 99 vertices). In healthy controls, $1.4 \pm$ $0.8 \mathrm{FP}$ clusters were generated in $21 / 59$ (35\%) individuals. Their size was 27.2 \pm 21.3 vertices (range: 3 - 66 vertices). Examples are presented in Figure 2.

To evaluate the efficacy of our two-step classification, we changed the threshold of the vertex-wise classifier from 0.87 to 0.94 and obtained on average $1.85 \pm$ $1.66 \mathrm{FP}$ clusters in healthy controls. This rate is comparable to the amount of FP clusters kept by the above cluster-wise classification. However, at this threshold, only 9/19 (47\%) lesions were detected. This demonstrates that the cluster-wise classification is an efficient step in the removal of FP, while most of the lesional clusters are maintained.

\section{Discussion}

The purpose of this study was to develop an automatic method for small FCD detection, a challenging and clinically valuable task that has not been addressed previously. We included features derived from textural and morphometric characteristics specific to small FCD lesions. To increase the sensitivity of our model, we introduced for the first time the concept of vertex-based analysis that allowed us to compute vertex-wise deviation from a group of healthy controls. We performed a two step classification combining vertex-wise and cluster-wise classification.

The vertex-wise classifier successfully identified $95 \%$ of small FCD lesions. Furthermore, the cluster-wise classifier efficiently removed false positive clusters, while retaining high detection rate of $68 \%$. This represents a 6.5 times higher detection rate that conventional radiological visual inspection, which allowed identifying only about $10 \%$ of the cases. 


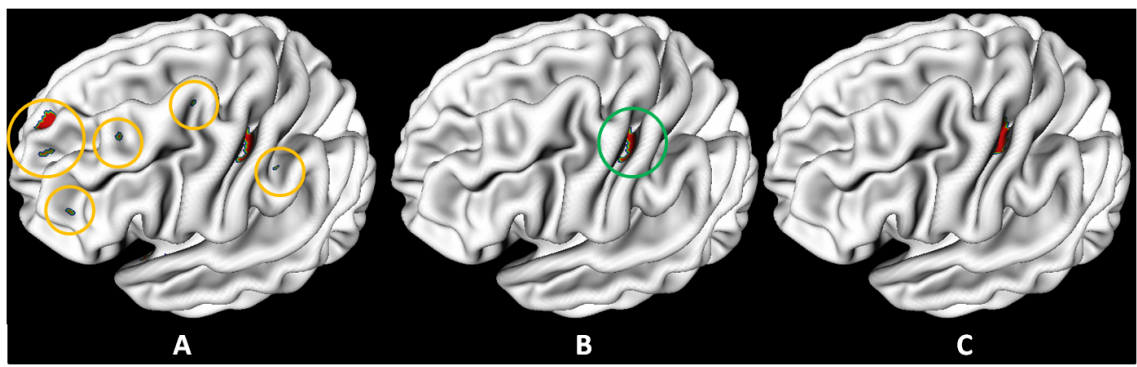

Fig. 3. A. Result of the vertex-wise classification. B. Result of the cluster-wise classification. C. Manual label. The vertex-wise classification step (A) successfully identified the FCD lesion, but generated FP clusters (yellow circles). However, they were suppressed by the cluster-wise classification step (B) and only the lesional cluster remained (green circle).

The average size of the false positive clusters was similar in patients and healthy controls. However, in the patients the cluster-wise classifier revealed some unusually large clusters with high lesional membership value located in areas distant from the primary FCD lesion. These clusters may indicate structurally abnormal regions, as previously suggested in a voxel-based study of FCD [6]. In some cases, due to hyperintense GM, part of the lesion may be classified as WM leading to a displacement of the WM surface. Thus, the gradient, measured on the WM surface, will be low because positioned within lesional GM compensating for the erroneous GM thinning.

This new surface-based analysis may become a useful clinical tool to assist the detection of subtle FCD lesions that are frequently overlooked by conventional means of analysis. We designed our protocol to attain high specificity, which is critical in the management of patients who undergo presurgical evaluation for medically intractable seizures.

\section{References}

1. Taylor, D.C., Falconer, M.A., Bruton, C.J., Corsellis, J.A.N.: Focal Dysplasia of the Cerebral Cortex in Epilepsy. J. Neurol. Neurosurg. Psychiatry 34, 369-387 (1971)

2. Sisodiya, S.M.: Surgery for Malformations of Cortical Development Causing Epilepsy. Brain 123, 1075-1091 (2000)

3. Tassi, L., Colombo, N., Garbelli, R., Francione, S., Lo, R.G., Mai, R., Cardinale, F., Cossu, M., Ferrario, A., Galli, C., Bramerio, M., Citterio, A., Spreafico, R.: Focal Cortical Dysplasia: Neuropathological Subtypes, EEG, Neuroimaging and Surgical Outcome. Brain 125, 1719-1732 (2002)

4. Antel, S.B., Collins, D.L., Bernasconi, N., Andermann, F., Shinghal, R., Kearney, R.E., Arnold, D.L., Bernasconi, A.: Automated Detection of Focal Cortical Dysplasia Lesions Using Computational Models of their MRI Characteristics and Texture Analysis. Neuroimage 19, 1748-1759 (2003) 
5. Wilke, M., Kassubek, J., Ziyeh, S., Schulze-Bonhage, A., Huppertz, H.J.: Automated Detection of Gray Matter Malformations Using Optimized Voxel-Based Morphometry: a Systematic Approach. Neuroimage 20, 330-343 (2003)

6. Colliot, O., Bernasconi, N., Khalili, N., Antel, S.B., Naessens, V., Bernasconi, A.: Individual Voxel-Based Analysis of Gray Matter in Focal Cortical Dysplasia. Neuroimage 29, 162-171 (2006)

7. Colliot, O., Antel, S.B., Naessens, V.B., Bernasconi, N., Bernasconi, A.: In Vivo Profiling of Focal Cortical Dysplasia on High-Resolution MRI with Computational Models. Epilepsia 47, 134-142 (2006)

8. Besson, P., Bernasconi, A.: Small FCD Lesions are Located at the Bottom of a Sulcus. Epilepsia 47, 16 (2006)

9. Kim, J.S., Singh, V., Lee, J.K., Lerch, J., Ad-Dab'bagh, Y., MacDonald, D., Lee, J.M., Kim, S.I., Evans, A.C.: Automated 3-D Extraction and Evaluation of the Inner and Outer Cortical Surfaces Using a Laplacian Map and Partial Volume Effect Classification. NeuroImage 27, 210-221 (2005)

10. Dale, A.M., Fischl, B., Sereno, M.I.: Cortical Surface-Based Analysis. I. Segmentation and Surface Reconstruction. Neuroimage 9, 179-194 (1999)

11. Salat, D.H., Buckner, R.L., Snyder, A.Z., Greve, D.N., Desikan, R.S.R., Busa, E., Morris, J.C., Dale, A.M., Fischl, B.: Thinning of the Cerebral Cortex in Aging. Cereb. Cortex 14, 721-730 (2004)

12. Lerch, J.P., Pruessner, J.C., Zijdenbos, A., Hampel, H., Teipel, S.J., Evans, A.C.: Focal Decline of Cortical Thickness in Alzheimer's Disease Identified by Computational Neuroanatomy. Cereb. Cortex 15, 995-1001 (2005)

13. Luders, E., Narr, K.L., Thompson, P.M., Rex, D.E., Jancke, L., Steinmetz, H., Toga, A.W.: Gender Differences in Cortical Complexity. Nat. Neurosci. 7, 799-800 (2004)

14. Thompson, P.M., Woods, R.P., Mega, M.S., Toga, A.W.: Mathematical/Computational Challenges in Creating Deformable and Probabilistic Atlases of the Human Brain. Hum. Brain Mapp. 9, 81-92 (2000)

15. Lyttelton, O., Boucher, M., Robbins, S., Evans, A.: An Unbiased Iterative Group Registration Template for Cortical Surface Analysis. NeuroImage 34, 1535-1544 (2007)

16. Sled, J.G., Zijdenbos, A.P., Evans, A.C.: A Nonparametric Method for Automatic Correction of Intensity Nonuniformity in MRI Data. IEEE Trans. Med. Imaging 17, 87-97 (1998)

17. Collins, D.L., Neelin, P., Peters, T.M., Evans, A.C.: Automatic 3D Intersubject Registration of MR Volumetric Data in Standardized Talairach Space. J. Comput. Assist. Tomogr. 18, 192-205 (1994)

18. Zijdenbos, A.P., Forghani, R., Evans, A.C.: Automatic Quantification of MS Lesions in 3D MRI Brain Data Sets: Validation of INSECT. In: Wells, W.M., Colchester, A.C.F., Delp, S.L. (eds.) MICCAI 1998. LNCS, vol. 1496, pp. 439-448. Springer, Heidelberg (1998)

19. Lerch, J.P., Evans, A.C.: Cortical Thickness Analysis Examined through Power Analysis and a Population Simulation. NeuroImage 24, 163-173 (2005)

20. Colliot, O., Mansi, T., Bernasconi, N., Naessens, V., Klironomos, D., Bernasconi, A.: Segmentation of Focal Cortical Dysplasia Lesions on MRI Using Level Set Evolution. Neuroimage 32, 1621-1630 (2006) 